\title{
Zero-forcing beamforming for physical layer security of energy harvesting wireless communications
}

Fengchao $\mathrm{Zhu}^{1}$, Feifei Gao ${ }^{1 *}$ and Minli Yao ${ }^{2}$

\begin{abstract}
In this paper, we consider the physical layer security for simultaneous wireless information and power transfer (SWIPT) in a multiple-input single-output (MISO) system that is consisted of three nodes: one transmitter with multiple antennas, one information decoding (ID) receiver with single antenna, and one energy harvesting (EH) receiver with single antenna. We propose a new zero-forcing based strategy that contains both the information beamforming and the energy beamforming, pointing to different receivers. To prevent the energy receiver from possibly eavesdropping the information, our target is to maximize the secrecy-rate of the ID receiver while at the same time maintaining a minimum required energy for the $\mathrm{EH}$ receiver. For the case that artificial noise is not used, the original non-convex problem can be directly converted into convex subproblems, where the closed-form optimal solutions are derived. For the case that artificial noise is used, the initial non-convex problem can be decomposed into two quasi-convex subproblems where closed-form solutions are derived, and the global optimal solutions are obtained with the aid of one-dimensional search. Simulations results demonstrate the trade-off between the maximum secret information rate and the transferred energy, which is characterized by the boundary of secret rate-energy (R-E) region.
\end{abstract}

Keywords: Physical layer security; Simultaneous wireless information and power transfer; Beamforming; Energy harvesting

\section{Introduction}

Since radio-frequency (RF) signals that carry information can, at the same time, be used for transporting energy, simultaneous wireless information and power transfer (SWIPT) is made possible in energy-constrained wireless networks and has become an interesting research area recently [1-5].

In [1], Varshney proposed a capacity-energy function for SWIPT, where a fundamental trade-off between the energy and the reliable information transmitted over a single noisy line is studied. In [2], Grover and Sahai extended the results of [1] to frequency-selective singleantenna additive white Gaussian noise (AWGN) channels, where a similar trade-off in frequency-domain is

\footnotetext{
*Correspondence: feifeigao@ieee.org

${ }^{1}$ Department of Automation, Tsinghua University, State Key Lab of Intelligent Technologies and Systems, Tsinghua National Laboratory for Information Science and Technology (TNList), No. 30 Shuang Qing Road, 100084 Beijing, P. R. China

Full list of author information is available at the end of the article
}

demonstrated. In [3], the robust case for SWIPT was studied using beamforming. In [4], Zhang revealed some fundamental issues in designing wireless multiple-input multiple-output (MIMO) systems to maximize the efficiency of SWIPT. Both information decoding (ID) receiver and energy harvesting $(\mathrm{EH})$ receiver that are separated or co-located were investigated in [4], and the optimal transmission strategy was derived to achieve different tradeoffs between maximal information rate and transferred energy. Most recently, in [5], the authors studied MIMO beamforming with partial channel state information (CSI) under energy harvesting constraints.

On the other hand, information-theoretic approach to guarantee secrecy was initiated by Wyner [6], where the concept of secrecy capacity was, for the first time, defined in degraded discrete memoryless wiretap channels. In [7], the results of [6] was generalized to a broadcast channel. Moreover, information secrecy in multiple access channels (MAC) was studied in [8-10], and information

\section{是 Springer}

(c) 2015 Zhu et al: licensee Springer. This is an Open Access article distributed under the terms of the Creative Commons Attribution License (http://creativecommons.org/licenses/by/4.0), which permits unrestricted use, distribution, and reproduction in any medium, provided the original work is properly credited. 
secrecy in single-input single-output (SISO) fading channels was also studied in [11-13]. To guarantee information secrecy using Wyner's model, the channel condition of the legitimate user is required to be better than that of the eavesdropper. In practice, however, this requirement may not be satisfied. Recently, an interesting way to achieve the secrecy using the aided noise was proposed in [14], where the information secrecy could be guaranteed even when the channel condition of the legitimate user is worse than that of the eavesdropper. Then the idea of aided noise were used in lots of works [15-22] where the physical layer security were studied. For example, [18-20] assume that transmitters cooperate to generate the aided noise, while $[15-17,21,22]$ require the aided noise spread in the null-space of the legitimate receiver's channel. Moreover, [23] studied the case where the noise covariance could take any spatial pattern. In addition, some other secure transmitting designs with the aided noise were also proposed aiming to provide the legitimate receiver with different quality-of-service (QoS), e.g., the SINR-based design [24] and the mean squared error (MSE)-based design [25].

The physical layer security for SWIPT system was first studied in [26], where secret information beamforming vector and artificial noise transmit covariance were designed. However, in [26], the closed-form solutions for information beamforming vector and energy beamforming vector cannot be derived. In this paper, we study information secrecy of SWIPT using zero-forcing beamforming which has lower computational complexity than the algorithm proposed in [26]. Remarkably, closed-form solutions are derived for both information beamforming vector and artificial noise (also used as energy) beamforming vector. The main contributions of this paper are summarized as follows:

1. For the feasibility problem, we obtain the closed-form solutions for energy beamforming vector, which can be derived with maximal-ratio-combining (MRC) principle.

2. For the conventional physical layer security problem without artificial noise, we first prove that the initial non-convex problem can be divided into two subproblems, based on different values of energy harvest requirements. Then, the closed-form solutions for the subproblems are derived.

3. For SWIPT with joint energy beamforming and information beamforming, we design zero-forcing beamforming for physical layer security. The optimal solutions for the non-convex problem is obtained using one-dimensional search. Remarkably, at each search step, closed-form solutions can be derived for all subproblems.
Notation: Vectors and matrices are boldface small and capital letters, respectively; the transpose, complex conjugate, Hermitian, inverse, and the pseudo-inverse of $A$ are denoted by $\boldsymbol{A}^{\mathrm{T}}, \boldsymbol{A}^{*}, \boldsymbol{A}^{\mathrm{H}}, \boldsymbol{A}^{-1}$, and $\boldsymbol{A}^{\dagger}$, respectively; $\operatorname{Tr}(\boldsymbol{A})$ and $A^{1 / 2}$ denote the trace and the square-root, respectively; $\boldsymbol{I}$ and $\mathbf{0}$ denote an identity matrix and an all-zero matrix, respectively, with appropriate dimensions; $\boldsymbol{A} \succeq \mathbf{0}$ and $\boldsymbol{A} \succ \mathbf{0}$ mean that $\boldsymbol{A}$ is positive semi-definite and positive definite, respectively; $\mathbb{E}[\cdot]$ denotes the statistical expectation; The distribution of a CSCG random variable with zero mean and variance $\sigma^{2}$ is denoted as $\mathcal{C N}\left(0, \sigma^{2}\right)$, and $\sim$ means 'distributed as'; $\mathbb{C}^{a \times b}$ denotes the space of $a \times b$ matrices with complex entries; $\|\mathbf{x}\|$ denotes the Euclidean norm of a vector $\mathbf{x}$; the unit-norm vector of a vector $\mathbf{x}$ is denoted as $\overrightarrow{\mathbf{x}}=\mathbf{x} /\|\mathbf{x}\|$; the quantity $\min (a, b)$ and $\max (a, b)$ represents the minimum and maximum between two real numbers.

\section{System model and problem formulation}

Let us consider a MISO system with one transmitter, one ID receiver, and one EH receiver, as shown in Figure 1. The transmitter is equipped with $M \geq 1$ antennas, while the ID receiver and the $\mathrm{EH}$ receiver are both equipped with single antenna. Both the information and the energy are transmitted over the same frequency band. Denote the baseband equivalent channels from the transmitter to the ID receiver and the $E H$ receiver as $\boldsymbol{h} \in \mathbb{C}^{M \times 1}$ and $\boldsymbol{g} \in \mathbb{C}^{M \times 1}$, respectively. We further assume that the channels are quasi-static fading while at each fading state, both $\boldsymbol{h}$ and $\boldsymbol{g}$ are known to the transmitter.

In case that the $\mathrm{EH}$ receiver may be an potential eavesdropper, the transmitter should adjust its strategy such that information secrecy of the ID receiver is guaranteed when operating in SWIPT mode. We then propose to use

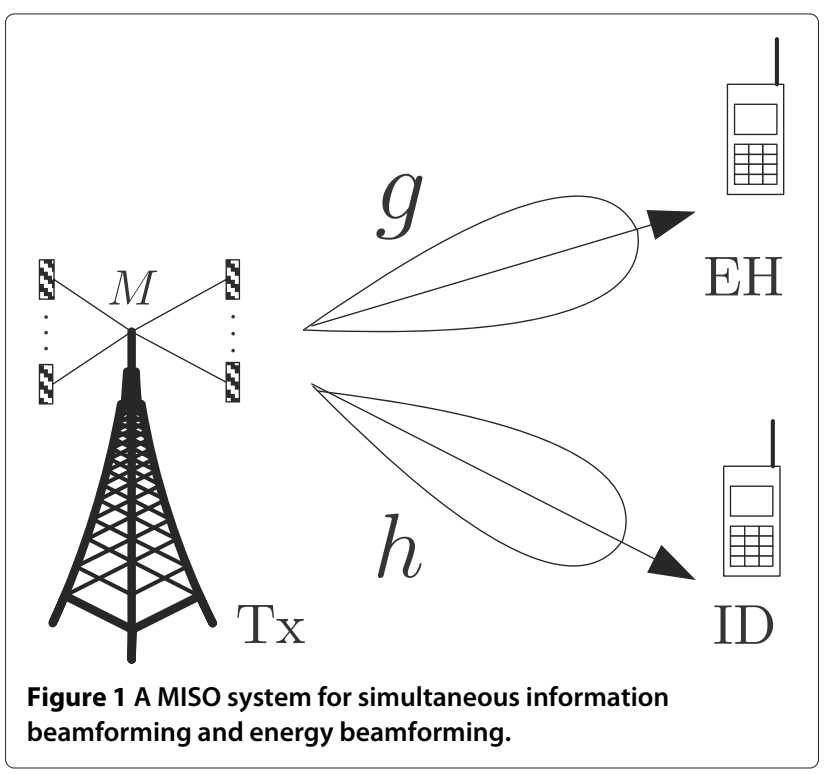


the aided noise to offer the energy to the $\mathrm{EH}$ receiver while at the same time preventing it from decoding the message. Meanwhile, the information beamforming will also be formulated to improve the signal quality at the ID receiver. Hence, the baseband transmitted signal can be expressed as:

$$
\boldsymbol{x}=\boldsymbol{s} v_{i}+\boldsymbol{w} v_{e}
$$

where $\boldsymbol{s} \in \mathbb{C}^{M \times 1}$ and $\boldsymbol{w} \in \mathbb{C}^{M \times 1}$ denote the information beamforming vector and the energy beamforming vector to be designed; $v_{i}$ is the CSCG random information bearing signal that is assumed to have the distribution $v_{i} \sim$ $\mathcal{C} \mathcal{N}(0,1)$. In order to provide the strongest disturbance to $\mathrm{EH}$ receiver, the energy-bearing signal should also be CSCG and has the distribution $v_{e} \sim \mathcal{C N}(0,1)$.

The signals received by the ID receiver and the $E H$ receiver are then given by:

$$
y_{i}=\boldsymbol{h}^{\mathrm{H}} \boldsymbol{x}+z_{i}, \quad y_{e}=\boldsymbol{g}^{\mathrm{H}} \boldsymbol{x}+z_{e}
$$

where $z_{i} \sim \mathcal{C N}(0,1)$ and $z_{e} \sim \mathcal{C N}(0,1)$ are the corresponding receiver noise.

The secrecy rate for the general non-degraded discrete memoryless wiretap channel is defined as [7]:

$$
C_{s} \triangleq \max _{p(\mathbf{u}, \boldsymbol{x})} I\left(\mathbf{u} ; y_{i}\right)-I\left(\mathbf{u} ; y_{e}\right)
$$

where $I\left(\mathbf{u} ; y_{i}\right)$ and $I\left(\mathbf{u} ; y_{e}\right)$ are the mutual information at the ID receiver and the $\mathrm{EH}$ receiver, respectively; $\mathbf{u}$ is an auxiliary variable satisfying the Markov relation $\mathbf{u} \rightarrow \boldsymbol{x} \rightarrow$ $\left(y_{i}, y_{e}\right) ; p(\mathbf{u}, \boldsymbol{x})$ is the joint distributions of $\mathbf{u}$ and $\boldsymbol{x}$ that satisfy the Markov relation.

Denoting $\boldsymbol{S}=\boldsymbol{s s}^{\mathrm{H}}$ and $\boldsymbol{W}=\boldsymbol{w} \boldsymbol{w}^{\mathrm{H}}$, the secrecy rate optimization problem ${ }^{\mathrm{a}}$ can be formulated as:

$$
\begin{aligned}
\text { PA : } \max _{\boldsymbol{S} \succeq \mathbf{0}, \boldsymbol{W} \succeq \mathbf{0}} \log _{2}\left(1+\frac{\boldsymbol{h}^{\mathrm{H}} \boldsymbol{S h}}{1+\boldsymbol{h}^{\mathrm{H}} \boldsymbol{W h}}\right)-\log _{2}\left(1+\frac{\boldsymbol{g}^{\mathrm{H}} \boldsymbol{S} \boldsymbol{g}}{1+\boldsymbol{g}^{\mathrm{H}} \boldsymbol{W} \boldsymbol{g}}\right) \\
\text { s.t. } \quad \operatorname{Tr}(\boldsymbol{S}+\boldsymbol{W}) \leq P \\
\boldsymbol{g}^{\mathrm{H}} \boldsymbol{S g}+\boldsymbol{g}^{\mathrm{H}} \boldsymbol{W} \boldsymbol{g}+1 \geq Q \\
\\
\operatorname{Rank}(\boldsymbol{S})=1, \quad \operatorname{Rank}(\boldsymbol{W})=1
\end{aligned}
$$

where $P$ is the transmit power limit and $Q$ is the energy harvesting $\operatorname{target}^{\mathrm{b}}$. PA is a very hard problem, since the objective function and the rank-one constraints are nonconvex functions. We then discuss PA in the following cases.

\section{The feasibility problem}

For the case that there is no ID receiver, Tx can use its whole power to satisfy the EH receiver's energy constraint in Equation 5. Then PA reduces to the feasibility problem, i.e., if the reduced PA is infeasible, PA will always be infeasible. The reduced PA can be simplified as:

$$
\begin{array}{cl}
\max _{\boldsymbol{W} \succeq \mathbf{0}} & 0 \\
\text { s.t. } & \operatorname{Tr}(\boldsymbol{W}) \leq P \\
& \boldsymbol{g}^{\mathrm{H}} \boldsymbol{W} \boldsymbol{g}+1 \geq Q .
\end{array}
$$

The maximum $Q$ can be obtained by solving the following problem:

$$
\begin{array}{cl}
\max _{\boldsymbol{W} \succeq \mathbf{0}} & \boldsymbol{g} \boldsymbol{W} \boldsymbol{g}^{\mathrm{H}}+1 \\
\text { s.t. } & \operatorname{Tr}(\boldsymbol{W}) \leq P .
\end{array}
$$

It is easily known that the maximum $Q$ can be obtained when the energy beamforming direction is the same as that of channel $\boldsymbol{g}$. Consequently, $Q_{\max }=P\|\boldsymbol{g}\|^{2}+1$ is obtained and the optimal energy beamforming vector $\boldsymbol{w}^{*}$ can be derived as $\boldsymbol{w}^{*}=\sqrt{P} \boldsymbol{g} /\|\boldsymbol{g}\|$. Therefore, the optimal energy beamforming vector is identical to that of the conventional MISO transmission with MRC principle. In the rest of this paper, we assume PA is always feasible (i.e., $Q \leq Q_{\max }$ is satisfied).

\section{Secure SWIPT without artificial noise}

For the secure SWIPT without artificial noise, PA can be simplified as:

$$
\begin{aligned}
\mathbf{P B}: \max _{\boldsymbol{S} \succeq \mathbf{0}} & \log _{2}\left(1+\boldsymbol{h}^{\mathrm{H}} \boldsymbol{S h}\right)-\log _{2}\left(1+\boldsymbol{g}^{\mathrm{H}} \boldsymbol{S g}\right) \\
\text { s.t. } & \operatorname{Tr}(\boldsymbol{S}) \leq P, \quad \boldsymbol{g}^{\mathrm{H}} \boldsymbol{S g}+1 \geq Q \\
& \operatorname{Rank}(\boldsymbol{S})=1 .
\end{aligned}
$$

It is easily known that PB in its current form is still neither convex nor concave. Fortunately, PB can be reformulated as a quasi-convex problem or a convex problem based on different energy harvesting limits $Q$; for both, closed-form solutions can be derived.

Lemma 1. For the secure SWIPT without artificial noise, if the energy harvesting constraint $1 \leq Q \leq \boldsymbol{g}^{\mathrm{H}} \boldsymbol{S}^{*} \boldsymbol{g}+1$, PB is equivalent to:

$$
\begin{gathered}
\mathbf{P B}-1: \max _{\boldsymbol{S} \succeq \mathbf{0}} \log _{2}\left(1+\boldsymbol{h}^{\mathrm{H}} \boldsymbol{S h}\right)-\log _{2}\left(1+\boldsymbol{g}^{\mathrm{H}} \boldsymbol{S g}\right) \\
\text { s.t. } \operatorname{Tr}(\boldsymbol{S}) \leq P, \quad \operatorname{Rank}(\boldsymbol{S})=1
\end{gathered}
$$

where $S^{*}$ is the optimal transmit covariance matrix of $\mathbf{P B}-1$. If $\boldsymbol{g} \boldsymbol{S}^{*} \boldsymbol{g}^{\mathrm{H}}+1<Q \leq Q_{\max }$, PB is equivalent to:

$$
\begin{aligned}
\mathbf{P B}-2: \max _{\boldsymbol{S} \succeq \mathbf{0}} & \log _{2}\left(1+\boldsymbol{h}^{\mathrm{H}} \boldsymbol{S h}\right)-\log _{2} Q \\
\text { s.t. } & \operatorname{Tr}(\boldsymbol{S}) \leq P, \quad \boldsymbol{g}^{\mathrm{H}} \boldsymbol{S g}+1=Q \\
& \operatorname{Rank}(\boldsymbol{S})=1 .
\end{aligned}
$$

The optimal information beamforming vector for $\mathbf{P B}-2$ can be written in the form of $\boldsymbol{s}=\eta_{\boldsymbol{s}} \overrightarrow{\boldsymbol{g}}+\xi_{\boldsymbol{s}} \overrightarrow{\boldsymbol{g}}_{\perp}$, where $\overrightarrow{\boldsymbol{g}}=\boldsymbol{g} /\|\boldsymbol{g}\|$ and $\overrightarrow{\boldsymbol{g}}_{\perp}=\boldsymbol{g}_{\perp} /\left\|\boldsymbol{g}_{\perp}\right\| ; \boldsymbol{g}_{\perp}=\left(\boldsymbol{I}-\overrightarrow{\boldsymbol{g}} \overrightarrow{\boldsymbol{g}}^{\mathrm{H}}\right) \boldsymbol{h}$ is the projection of $\boldsymbol{h}$ onto the null space of $\boldsymbol{g} ; \eta_{\boldsymbol{s}}$ and $\xi_{\boldsymbol{s}}$ are complex weights. 
Proof. Assuming $S^{*}$ is the optimal transmitting covariance matrix of $\mathbf{P B}-1$, we know that the energy harvested at $\mathrm{EH}$ receiver can be expressed as $Q^{*}=\boldsymbol{g}^{\mathrm{H}} \boldsymbol{S}^{*} \boldsymbol{g}+1$. If $Q^{*} \geq Q$, it is easily known that $\mathbf{P B}$ is not limited by the energy harvesting constraint. Therefore, $S^{*}$ is also optimal for $\mathbf{P B}$ when $Q^{*} \geq Q$.

If $Q>g^{\mathrm{H}} \boldsymbol{S}^{*} \boldsymbol{g}+1$, it follows that $\mathbf{P B}$ is limited by the the energy harvesting constraint. Then, we prove that in PB at the optimal point, $\boldsymbol{g}^{\mathrm{H}} \boldsymbol{S}^{*} \boldsymbol{g}+1=Q$ must be satisfied by contradiction. Assuming the optimal $S^{*}$ for PB satisfies $\boldsymbol{g}^{\mathrm{H}} \boldsymbol{S}^{*} \boldsymbol{g}+1>Q$, it corresponds to the case of $\mathbf{P B}-1$, which contradicts the fact that $Q>\boldsymbol{g}^{\mathrm{H}} \boldsymbol{S}^{*} \boldsymbol{g}+1$ must be satisfied in this case. From Equation 13, we know that at the optimal point, there is $\boldsymbol{g}^{\mathrm{H}} \boldsymbol{S}^{*} \boldsymbol{g}+1=Q$. Substituting $\boldsymbol{g}^{\mathrm{H}} \boldsymbol{S}^{*} \boldsymbol{g}+1=Q$ into $\mathbf{P B}$, we know that for the case of $Q>\boldsymbol{g}^{\mathrm{H}} \boldsymbol{S}^{*} \boldsymbol{g}+1, \mathbf{P B}$ and $\mathbf{P B}-2$ have the same optimal solutions.

Aiming to prove that there is an optimal structure for the beamforming vector for $\mathbf{P B}-2$, we first prove that the optimal transmit covariance is rank-one. It is easily shown that $\mathbf{P B}-2$ has the same optimal information transmit covariance as the following problem:

$$
\begin{aligned}
\mathbf{P B}-3: \max _{\boldsymbol{S} \succeq \mathbf{0}} & \operatorname{Tr}(\mathbf{H} \boldsymbol{S}) \\
\text { s.t. } & \operatorname{Tr}(\boldsymbol{S}) \leq P \\
& \operatorname{Tr}(\boldsymbol{G S})+1=Q \\
& \operatorname{Rank}(\boldsymbol{S})=1,
\end{aligned}
$$

where $\mathbf{H}=\boldsymbol{h}^{\mathrm{H}}$ and $\boldsymbol{G}=\boldsymbol{g} \boldsymbol{g}^{\mathrm{H}}$. After dropping the rank-1 constraint in Equation 23, the Lagrange [27] of $\mathbf{P B}-3$ is defined as:

$$
\begin{aligned}
\mathcal{L}(\boldsymbol{S}, \alpha, \beta)= & \operatorname{Tr}(\mathbf{H} \boldsymbol{S})-\alpha(\operatorname{Tr}(\boldsymbol{S})-P) \\
& -\beta(\operatorname{Tr}(\mathbf{G S})+1-Q) \\
= & \operatorname{Tr}(\Phi \boldsymbol{S})+(\alpha P-\beta+\beta Q),
\end{aligned}
$$

where $\Phi=\mathbf{H}-\alpha \boldsymbol{I}-\beta \boldsymbol{G} ; \alpha$ and $\beta$ denote the dual variables associated with the constraints in Equations 21 and 22, respectively. Then, the Lagrangian dual function [27] of $\mathbf{P B}-3$ is defined as:

$$
\zeta(\alpha \geq 0, \beta)=\max _{\boldsymbol{S} \succeq \mathbf{0}} \mathcal{L}(\boldsymbol{S}, \alpha, \beta),
$$

which must have a bounded optimal value. The dual problem [27] of $\mathbf{P B}-3$ is defined as:

$$
\min _{\alpha, \beta} \max _{\boldsymbol{S} \succeq \mathbf{0}} \mathcal{L}(\boldsymbol{S}, \alpha, \beta) .
$$

The Lagrangian dual problem for $\mathbf{P B}-3$ can be further expressed as:

$$
\begin{aligned}
& \min _{\alpha, \beta} \alpha P-\beta+\beta Q \\
& \text { s.t. }-\Phi \succeq \mathbf{0}, \quad \alpha \geq 0 .
\end{aligned}
$$

The Karush-Kuhn-Tucker (KKT) conditions [27] related to $S$ can be formulated as:

$$
\begin{aligned}
\boldsymbol{H}-\alpha^{*} \boldsymbol{I}-\beta^{*} \boldsymbol{G} & =\Phi^{*}, \\
\Phi^{*} \boldsymbol{S}^{*} & =\mathbf{0},
\end{aligned}
$$

where $\alpha^{*} \geq 0, \beta^{*}$ and $\Phi^{*} \preceq 0$ denote the corresponding optimal dual variables.

Lemma 1.1. The optimal dual variable $\alpha^{*}$ satisfies $\alpha^{*}>0$.

Proof. Assuming $\alpha^{*}=0$, Equation 29 can be reexpressed as:

$$
\boldsymbol{H}-\beta^{*} \boldsymbol{G}=\Phi^{*} .
$$

Using the constraint $\Phi^{*} \preceq \mathbf{0}$, for any $\boldsymbol{x} \neq \mathbf{0}$, we have $\boldsymbol{x}^{\mathrm{H}} \Phi^{*} \boldsymbol{x} \leq 0$. Assuming $\boldsymbol{x}^{\mathrm{H}} \boldsymbol{G} \boldsymbol{x}=0$, from Equation 31 we obtain:

$$
\boldsymbol{x}^{\mathrm{H}} \Phi^{*} \boldsymbol{x}=\boldsymbol{x}^{\mathrm{H}} \boldsymbol{H} \boldsymbol{x} \leq 0 .
$$

Since $\boldsymbol{H} \succeq \mathbf{0}$, there is $\boldsymbol{x}^{\mathrm{H}} \boldsymbol{H} \boldsymbol{x} \geq 0$. Thus, we know $\boldsymbol{x}^{\mathrm{H}} \boldsymbol{H} \boldsymbol{x}=$ 0 must hold. It requires that any $\boldsymbol{x} \neq \mathbf{0}$ lies in the null space of $\boldsymbol{G}$ must also be in the null space of $\boldsymbol{H}$. However, it cannot be true since $\boldsymbol{h}$ and $\boldsymbol{g}$ are independent and randomly generated vectors and should be linearly independent. Consequently, $\alpha^{*}>0$ must hold in this case, which completes the proof of Lemma 1.1.

Since $\alpha^{*}>0$, assuming $\beta^{*} \geq 0$, from Equation 29 it follows that $-\alpha^{*} \boldsymbol{I}-\beta^{*} \boldsymbol{G}$ has a full rank $M$. Thus, from Equation 29, we obtain $\operatorname{Rank}\left(\Phi^{*}\right) \geq M-1$. From Equation 30, we know:

$$
\operatorname{Rank}\left(\Phi^{*}\right)+\operatorname{Rank}\left(S^{*}\right) \leq M .
$$

Consequently, we obtain $\operatorname{Rank}\left(\boldsymbol{S}^{*}\right) \leq 1$. Assuming $\beta^{*}<0$, we will draw the contradiction as follows. Consider first the problem in Equation 25 of maximizing the Lagrangian over $S$ with fixed $\alpha$ and $\beta$. Discarding the constant terms associated with $\alpha$ and $\beta$, problem in Equation 25 can be equivalently rewritten as:

$$
\max _{\boldsymbol{S} \succeq \mathbf{0}} \operatorname{Tr}((\boldsymbol{H}-\alpha \boldsymbol{I}-\beta \boldsymbol{G}) \boldsymbol{S}) .
$$

It is easily shown that problem in Equation 34 is unbounded when $\beta \rightarrow-\infty$. Thus, $\beta^{*}<0$ cannot be true. From the above discussions, we know that the optimal $S^{*}$ for $\mathbf{P B}-2$ is indeed rank-1. Thus, $\boldsymbol{S}$ can be expressed as $\boldsymbol{S}=\boldsymbol{s} \boldsymbol{s}^{\mathrm{H}}$, where $\boldsymbol{s}$ is the information beamforming vector. PB -2 can be equivalently reformulated as:

$$
\begin{aligned}
\mathbf{P B}-4: \max _{\boldsymbol{s}} & \log _{2}\left(1+\left\|\boldsymbol{h}^{\mathrm{H}} \boldsymbol{s}\right\|^{2}\right)-\log _{2} Q \\
\text { s.t. } & \|\boldsymbol{s}\|^{2} \leq P, \\
& \left\|\boldsymbol{g}^{\mathrm{H}} \boldsymbol{s}\right\|^{2}+1=Q .
\end{aligned}
$$

Next, let us prove that there exist an optimal structure for the information beamforming vector for $\mathbf{P B}-4$. We can first assume there is $\overrightarrow{\boldsymbol{k}}$ that satisfies $\overrightarrow{\boldsymbol{k}}^{\mathrm{H}} \overrightarrow{\boldsymbol{g}}=0$. Then, 
the optimal information beamforming vector $s^{*}$ for PC -4 can be expressed as $\boldsymbol{s}^{*}=\eta_{\boldsymbol{s}^{*}} \overrightarrow{\boldsymbol{g}}+\xi_{s^{*}} \overrightarrow{\boldsymbol{k}}$. Since $\overrightarrow{\boldsymbol{g}}_{\perp}^{\mathrm{H}} \overrightarrow{\boldsymbol{g}}=0$ and $\left\|\boldsymbol{h}^{\mathrm{H}} \overrightarrow{\boldsymbol{g}}_{\perp}\right\| \geq\left\|\boldsymbol{h}^{\mathrm{H}} \overrightarrow{\boldsymbol{k}}\right\|$, replacing $\overrightarrow{\boldsymbol{k}}$ with $\overrightarrow{\boldsymbol{g}}_{\perp}$ does not influence the equality constraint in Equation 36 in PC -4 but always helps to improve the value of Equation 35. Thus, the optimal information beamforming vector can be expressed as $\boldsymbol{s}^{*}=\eta_{\boldsymbol{s}^{*}} \overrightarrow{\boldsymbol{g}}+\xi_{s^{*}} \overrightarrow{\boldsymbol{g}}_{\perp}$.

Lemma 1 is thus proved.

Lemma 1 implies that problem PB can be divided into two subproblems depending on $Q$. The optimal information beamforming vector can be derived. To summarize, the following theorem is established.

Theorem 1. For the secure SWIPT without artificial noise, if $1 \leq Q \leq \boldsymbol{g}^{\mathrm{H}} \boldsymbol{S}^{*} \boldsymbol{g}+1$ which corresponding to $\mathbf{P B}-1$, the optimal information beamforming vector can be obtained as:

$$
\boldsymbol{s}^{*}=\frac{\sqrt{P}\left(\boldsymbol{I}+P \boldsymbol{g} \boldsymbol{g}^{\mathrm{H}}\right)^{-1 / 2} \mathbf{e}}{\left\|\left(\boldsymbol{I}+\boldsymbol{P g}^{\mathrm{H}}\right)^{-1 / 2} \mathbf{e}\right\|}
$$

where $\mathbf{e}$ is any scaled version of the eigenvector of $\mathbf{Z}$ corresponding to its largest eigenvalue and $\mathbf{Z}$ is defined as:

$$
\mathbf{Z}=\left(\boldsymbol{I}+P \boldsymbol{g} \boldsymbol{g}^{\mathrm{H}}\right)^{-1 / 2}\left(\boldsymbol{I}+P \boldsymbol{h} \boldsymbol{h}^{\mathrm{H}}\right)\left(\boldsymbol{I}+P \boldsymbol{g} \boldsymbol{g}^{\mathrm{H}}\right)^{-1 / 2} .
$$

If $\boldsymbol{g}^{\mathrm{H}} \boldsymbol{S}^{*} \boldsymbol{g} \leq Q \leq Q_{\max }$ which corresponding to $\mathbf{P B}-2$, the optimal information beamforming vector can be expressed as $\boldsymbol{s}^{*}=\eta_{s}^{*} \overrightarrow{\boldsymbol{g}}+\xi_{s}^{*} \overrightarrow{\boldsymbol{g}}_{\perp}$ where $\eta_{\boldsymbol{s}}^{*}$ and $\xi_{\boldsymbol{s}}^{*}$ can be derived as following:

$$
\eta_{s}^{*}=\frac{\sqrt{Q-1}}{\|\boldsymbol{g}\|} \frac{\eta_{\boldsymbol{h}}}{\left\|\eta_{\boldsymbol{h}}\right\|}, \quad \xi_{\boldsymbol{s}}^{*}=\sqrt{P-\frac{Q-1}{\|\boldsymbol{g}\|^{2}}} \frac{\xi_{\boldsymbol{h}}}{\left\|\xi_{\boldsymbol{h}}\right\|} .
$$

Proof. When $1 \leq Q \leq \boldsymbol{g}^{\mathrm{H}} \boldsymbol{S}^{*} \boldsymbol{g}+1$, we know that $\mathbf{P B}-1$ has the same optimal solutions as:

$$
\begin{aligned}
\max _{\boldsymbol{S} \succeq \mathbf{0}} & \frac{1+\boldsymbol{h}^{\mathrm{H}} \boldsymbol{h}}{1+\boldsymbol{g}^{\mathrm{H}} \boldsymbol{S g}} \\
\text { s.t. } & \operatorname{Tr}(\boldsymbol{S}) \leq \theta P,
\end{aligned}
$$

where the optimal information beamforming vector can be derived as [28].

When $\boldsymbol{g}^{\mathrm{H}} \boldsymbol{S}^{*} \boldsymbol{g} \leq Q \leq Q_{\max }$, we can first express $\boldsymbol{h}$ as $\boldsymbol{h}=\eta_{\boldsymbol{h}} \overrightarrow{\boldsymbol{g}}+\xi_{\boldsymbol{h}} \overrightarrow{\boldsymbol{g}}_{\perp}$. Then, it follows that $\mathbf{P B}-2$ has the same optimal solutions as:

$$
\begin{aligned}
\max & \log _{2}\left(1+\left\|\eta_{\boldsymbol{h}}^{\mathrm{H}} \eta_{\boldsymbol{s}}+\xi_{\boldsymbol{h}}^{\mathrm{H}} \xi_{\boldsymbol{s}}\right\|^{2}\right)-\log _{2}(Q) \\
\text { s.t. } & \left\|\eta_{\boldsymbol{s}}\right\|^{2}+\left\|\xi_{\boldsymbol{s}}\right\|^{2} \leq P \\
& \|\boldsymbol{g}\|^{2}\left\|\eta_{s}\right\|^{2}+1=Q
\end{aligned}
$$

where the closed-form solutions can be easily derived by using standard geometry.

The proof is thus completed.

\section{Zero-forcing-based joint information and energy beamforming}

When artificial noise is used, we should design joint information and energy beamforming. Since PA is very hard to be solved, we let the information beamforming vector $\boldsymbol{s}$ be in the null space of $\boldsymbol{g}$ (i.e., $\boldsymbol{g}^{\mathrm{H}} \boldsymbol{S g}=0$ ). Thus, the EH receiver cannot receive the information signal, and the energy harvest constraint is satisfied by energy beamforming. Let us define an auxiliary variable $\theta$ as the proportion of the transmit power assigned to the energy beamforming. Then, PA can be then reformulated as:

$$
\begin{aligned}
\mathbf{P C}: & \max _{\boldsymbol{S} \succeq \mathbf{0}, \boldsymbol{W} \succeq \mathbf{0}} \log _{2}\left(1+\frac{\boldsymbol{h}^{\mathrm{H}} \boldsymbol{S} \boldsymbol{h}}{1+\boldsymbol{h}^{\mathrm{H}} \boldsymbol{W h}}\right) \\
\text { s.t. } & \operatorname{Tr}(\boldsymbol{S}) \leq(1-\theta) P, \operatorname{Tr}(\boldsymbol{W}) \leq \theta P \\
& \boldsymbol{g}^{\mathrm{H}} \boldsymbol{W} \boldsymbol{g}+1 \geq Q \\
& \boldsymbol{g}^{\mathrm{H}} \boldsymbol{S} \boldsymbol{g}=0,0 \leq \theta \leq 1 \\
& \operatorname{Rank}(\boldsymbol{S})=1, \quad \operatorname{Rank}(\boldsymbol{W})=1 .
\end{aligned}
$$

By using primal decomposition, $\mathbf{P C}$ can be divided into two subproblems. The result is implied by the following proposition.

Proposition 1. PC has the same optimal solutions $S^{*}$ and $W^{*}$ as that of:

$$
\begin{aligned}
\mathbf{P D}: \max & f_{1}(\theta) f_{2}(1-\theta) \\
\text { s.t. } & 0 \leq \theta \leq 1,
\end{aligned}
$$

where $f_{1}(\theta)$ is defined as:

$$
\begin{aligned}
f_{1}(\theta)=\max _{\boldsymbol{W} \geq \mathbf{0}} & \frac{1}{1+\boldsymbol{h}^{\mathrm{H}} \boldsymbol{W h}} \\
\text { s.t. } & \operatorname{Tr}(\boldsymbol{W}) \leq \theta P \\
& \boldsymbol{g}^{\mathrm{H}} \boldsymbol{W g}+1 \geq Q \\
& \operatorname{Rank}(\boldsymbol{W})=1,
\end{aligned}
$$

and $f_{2}(1-\theta)$ is defined as:

$$
\begin{aligned}
f_{2}(1-\theta)=\max _{\boldsymbol{S} \geq \mathbf{0}} & \boldsymbol{h}^{\mathrm{H}} \boldsymbol{S} \\
\text { s.t. } & \operatorname{Tr}(\boldsymbol{S}) \leq(1-\theta) P \\
& \boldsymbol{g}^{\mathrm{H}} \boldsymbol{S g}=0 \\
& \operatorname{Rank}(\boldsymbol{S})=1 .
\end{aligned}
$$

Proof. Since the objective function of $\mathbf{P C}$ can be expressed as the multiplication of two parts, primal decomposition [27] can be used to divide PC into two subproblems. The proof is completed.

To obtain the closed-form solution for $f_{1}(\theta)$, the following two lemmas are needed.

Lemma 2. The optimal $W$ for $f_{1}(\theta)$ must satisfy $\operatorname{Rank}(W)=1$. 
Proof. We first relax $f_{1}(\theta)$ by dropping the rank-one constraint that $\operatorname{Rank}(W)=1$. Then, the same optimal $W$ for $f_{1}(\theta)$ can be obtained by solving the following problem:

$$
\begin{aligned}
f_{1}^{\prime}(\theta)=\min & \boldsymbol{h}^{\mathrm{H}} \boldsymbol{W} \boldsymbol{h} \\
\text { s.t. } & \operatorname{Tr}(\boldsymbol{W}) \leq \theta P \\
& \boldsymbol{g}^{\mathrm{H}} \boldsymbol{W} \boldsymbol{g}+1 \geq Q \\
& \boldsymbol{W} \succeq \mathbf{0} .
\end{aligned}
$$

The KKT conditions [27] for $f_{1}^{\prime}(\theta)$ can be formulated as:

$$
\begin{aligned}
\boldsymbol{h h}^{\mathrm{H}}+\lambda \boldsymbol{I} & =\mu \boldsymbol{g g}^{\mathrm{H}}+\Psi \\
\lambda(\operatorname{Tr}(\boldsymbol{W})-\theta P) & =0 \\
\mu\left(\boldsymbol{g} \boldsymbol{g}^{\mathrm{H}}-Q+1\right) & =0 \\
\operatorname{Tr}(\Psi \mathbf{W}) & =0
\end{aligned}
$$

where $\lambda \geq 0, \mu \geq 0$ and $\Psi \geq 0$ are the Lagrange multipliers associated with the constraints in Equation 62 to Equation 64, respectively.

When $\lambda=0$, it follows from Equation 65 that $g$ must be in parallel to $\boldsymbol{h}$. Consequently, it is easily shown in $f_{1}^{\prime}(\theta)$ that the optimal $\boldsymbol{W}$ is $\boldsymbol{W}=(Q-1) \boldsymbol{h} \boldsymbol{h}^{\mathrm{H}} /\left\|\boldsymbol{g}^{\mathrm{H}} \boldsymbol{h}\right\|^{2}$ and thus $\operatorname{Rank}(\boldsymbol{W})=1$.

When $\lambda>0$, it follows from Equation 65 that the left side has a full rank of $M$. while the right side of Equation 65 must also have a full rank of $M$. Consequently, we obtain $\operatorname{Rank}(\Psi) \geq M-1$. From Equation 68 , we obtain $\operatorname{Rank}(\boldsymbol{W})+\operatorname{Rank}(\Psi) \leq M$. Hence, there must be $\operatorname{Rank}(W) \leq 1$ and the proof is completed.

Lemma 3. For $f_{1}(\theta)$, the range of the optimal power allocation ratio $\theta$ must satisfy:

$$
\frac{Q-1}{P\|\boldsymbol{g}\|^{2}} \leq \theta \leq \frac{Q-1}{P\left\|\left(\boldsymbol{I}-\overrightarrow{\boldsymbol{h}} \overrightarrow{\boldsymbol{h}}^{\mathrm{H}}\right) \boldsymbol{g}\right\|^{2}},
$$

where $\overrightarrow{\boldsymbol{h}}=\boldsymbol{h} /\|\boldsymbol{h}\|$ is the unit-norm vector of the ID receiver's channel.

Proof. Since the optimal transmit covariance is indeed rank-1, we know that $W$ can be expressed as $W=$ $\boldsymbol{w} \boldsymbol{w}^{\mathrm{H}}$. To satisfy the energy harvesting constraint of $f_{1}(\theta)$, $\left\|\boldsymbol{g}^{\mathrm{H}} \boldsymbol{w}\right\|^{2}+1 \geq Q$ should be guaranteed. For any energy beamforming vector $\boldsymbol{w}$, there must be:

$$
Q \leq\left\|\boldsymbol{g}^{\mathrm{H}} \boldsymbol{w}\right\|^{2}+1 \leq\|\boldsymbol{g}\|^{2}\|\boldsymbol{w}\|^{2}+1 .
$$

Moreover, the transmit power constraint in Equation 62 can be reformulated as"

$$
\|\boldsymbol{w}\|^{2} \leq \theta P .
$$

From Equations 70 and 71, there is:

$$
\theta \geq \frac{Q-1}{P\|\boldsymbol{g}\|^{2}} .
$$

On the other hand, since $\boldsymbol{W} \succeq \mathbf{0}$, there must be $\boldsymbol{h}^{\mathrm{H}} \boldsymbol{W} \boldsymbol{h} \geq 0$. It is easily shown that the minimum value of Equation 61 is equal to zero when the energy beamforming vector $\boldsymbol{w}$ lies in the null space of the ID receiver's channel vector $\boldsymbol{h}$, i.e., $\boldsymbol{h}^{\mathrm{H}} \boldsymbol{w}=0$. Then, let us assume that $\boldsymbol{h}^{\mathrm{H}} \boldsymbol{w}=0$. It follows from Equation 63 that:

$$
Q \leq\left\|\boldsymbol{g}^{\mathrm{H}} \boldsymbol{w}\right\|^{2}+1 \leq\left\|\left(\boldsymbol{I}-\overrightarrow{\boldsymbol{h}} \overrightarrow{\boldsymbol{h}}^{\mathrm{H}}\right) \boldsymbol{g}\right\|^{2}\|\boldsymbol{w}\|^{2}+1
$$

must hold. From Equations 71 and 73, there must be:

$$
\theta \geq(Q-1) /\left(P\left\|\left(\boldsymbol{I}-\overrightarrow{\boldsymbol{h}} \overrightarrow{\boldsymbol{h}}^{\mathrm{H}}\right) \boldsymbol{g}\right\|^{2}\right) .
$$

Then, it is easily shown that for any $\theta$ that $\theta>(Q-$ 1) $/\left(P\left\|\left(\boldsymbol{I}-\overrightarrow{\boldsymbol{h}} \overrightarrow{\boldsymbol{h}}^{\mathrm{H}}\right) \boldsymbol{g}\right\|^{2}\right)$, we can always find a smaller $\theta^{\prime}=$ $(Q-1) /\left(P\left\|\left(\boldsymbol{I}-\overrightarrow{\boldsymbol{h}} \overrightarrow{\boldsymbol{h}}^{\mathrm{H}}\right) \boldsymbol{g}\right\|^{2}\right)$ that maintains $\boldsymbol{h}^{\mathrm{H}} \boldsymbol{W} \boldsymbol{h}=0$. Meanwhile, the saved power can then be used for information beamforming to obtain a bigger secret rate. Consequently, the optimal power allocation ratio should not bigger than $(Q-1) /\left(P\left\|\left(\boldsymbol{I}-\overrightarrow{\boldsymbol{h}} \overrightarrow{\boldsymbol{h}}^{\mathrm{H}}\right) \boldsymbol{g}\right\|^{2}\right)$. And the proof is completed.

Lemma 3 implies that for PD, the power allocation ratio should be limited to a subset of $0 \leq \theta \leq 1$. The subset limitation is significant for complexity reduction in onedimensional search. In fact, if $\|\boldsymbol{g}\|^{2}=\left\|\left(\boldsymbol{I}-\overrightarrow{\boldsymbol{h}} \overrightarrow{\boldsymbol{h}}^{\mathrm{H}}\right) \boldsymbol{g}\right\|^{2}$, we can always obtain the optimal solutions without using one-dimensional search.

Using lemma 2 and lemma 3, we know that $f_{1}(\theta)$ has the same optimal information beamforming vector as:

$$
\begin{aligned}
\text { PD-1 }: \min & \left\|\boldsymbol{h}^{\mathrm{H}} \boldsymbol{w}\right\|^{2} \\
\text { s.t. } & \|\boldsymbol{w}\|^{2} \leq \theta P \\
& \left\|\boldsymbol{g}^{\mathrm{H}} \boldsymbol{w}\right\|^{2}+1 \geq Q .
\end{aligned}
$$

The optimal $\boldsymbol{w}$ for PD-1 can be expressed in the form of $\alpha_{\boldsymbol{w}} \overrightarrow{\boldsymbol{h}}+\beta_{\boldsymbol{w}} \overrightarrow{\boldsymbol{h}}_{\perp}$ where $\overrightarrow{\boldsymbol{h}}=\boldsymbol{h} /\|\boldsymbol{h}\|$ and $\overrightarrow{\boldsymbol{h}}_{\perp}=\boldsymbol{h}_{\perp} /\left\|\boldsymbol{h}_{\perp}\right\|$; $\boldsymbol{h}_{\perp}=\left(\boldsymbol{I}-\overrightarrow{\boldsymbol{h}} \overrightarrow{\boldsymbol{h}}^{\mathrm{H}}\right) \boldsymbol{g}$ is the projection of $\boldsymbol{g}$ onto the null space of $\boldsymbol{h} ; \alpha_{\boldsymbol{w}}$ and $\beta_{\boldsymbol{w}}$ are complex weights. The proof is similar as lemma 1 and is omitted here for brief. Then, we can let $\boldsymbol{g}=\alpha_{\boldsymbol{g}} \overrightarrow{\boldsymbol{h}}+\beta_{\boldsymbol{g}} \overrightarrow{\boldsymbol{h}}_{\perp}$. PD -1 can be equivalently reformulated as:

$$
\begin{aligned}
\text { PD-2 }: \min & \left\|\alpha_{\boldsymbol{w}}\right\|^{2}\|\boldsymbol{h}\|^{2} \\
\text { s.t. } & \left\|\alpha_{\boldsymbol{w}}\right\|^{2}+\left\|\beta_{\boldsymbol{w}}\right\|^{2} \leq \theta P \\
& \left\|\alpha_{w}^{\mathrm{H}} \alpha_{\boldsymbol{g}}+\beta_{w}^{\mathrm{H}} \beta_{\boldsymbol{g}}\right\|^{2}+1 \geq Q .
\end{aligned}
$$

To derive the closed-form solution for PD-2, the following lemma is needed.

Lemma 4. The optimal $\alpha_{w}$ and $\beta_{w}$ for PD-2 must satisfy:

$$
\begin{aligned}
\left\|\alpha_{w}\right\|^{2}+\left\|\beta_{w}\right\|^{2} & =\theta P, \\
\left\|\alpha_{w}^{\mathrm{H}} \alpha_{\boldsymbol{g}}+\beta_{w}^{\mathrm{H}} \beta_{\boldsymbol{g}}\right\|^{2}+1 & =Q .
\end{aligned}
$$

Proof. It is easily known that when $\left\|\alpha_{w}\right\|^{2}+\left\|\beta_{w}\right\|^{2}<\theta P$, we can always decrease $\theta$ to improve the power allocated for the information beamforming to obtain a bigger secret rate. Thus, at the optimal point, there must be $\left\|\alpha_{w}\right\|^{2}+$ $\left\|\beta_{\boldsymbol{w}}\right\|^{2}=\theta P$. 
Next, let us prove that Equation 82 is satisfied using the result of lemma 3.

Case 1: $\theta=(Q-1) /\left(P\|\boldsymbol{g}\|^{2}\right)$ : It follows from Equation 70 that $\boldsymbol{w}$ is parallel to $\boldsymbol{g}$. Thus, there must be $\beta_{\boldsymbol{w}}=\mathbf{0}$. Then PD-2 can be simplified as:

$$
\begin{array}{cl}
\min & \left\|\alpha_{\boldsymbol{w}}\right\|^{2}\|\boldsymbol{h}\|^{2} \\
\text { s.t. } & \left\|\alpha_{\boldsymbol{w}}\right\|^{2} \leq \theta P \\
& \left\|\alpha_{\boldsymbol{w}}^{\mathrm{H}} \alpha_{\boldsymbol{g}}\right\|^{2}+1 \geq Q
\end{array}
$$

where it is easy to observe that $\left\|\alpha_{w}^{\mathrm{H}} \alpha_{g}\right\|^{2}+1=Q$ must be satisfied.

Case 2: $\theta=(Q-1) /\left(P\left\|\left(\boldsymbol{I}-\overrightarrow{\boldsymbol{h}} \overrightarrow{\boldsymbol{h}}^{\mathrm{H}}\right) \boldsymbol{g}\right\|^{2}\right)$ : It follows from Equation 73 that $\boldsymbol{w}$ is orthogonal to $g$. Thus, there must be $\alpha_{\boldsymbol{w}}=\mathbf{0}$. Then PD -2 can be simplified as:

$$
\begin{array}{cl}
\min & 0 \\
\text { s.t. } & \left\|\beta_{w}\right\|^{2} \leq \theta P \\
& \left\|\beta_{w}^{\mathrm{H}} \beta_{g}\right\|^{2}+1 \geq Q .
\end{array}
$$

Using the proof of lemma 3, we know that at the optimal point $\left\|\beta_{w}^{\mathrm{H}} \beta_{g}\right\|^{2}+1=Q$ must be satisfied to save the transmit power.

Case 3: $(Q-1) /\left(P\|\boldsymbol{g}\|^{2}\right)<\theta<(Q-1) /(P \|(I-$ $\left.\left.\overrightarrow{\boldsymbol{h}} \overrightarrow{\boldsymbol{h}}^{\mathrm{H}}\right) \boldsymbol{g} \|^{2}\right)$ : There must be $\left\|\alpha_{\boldsymbol{w}}\right\|>0$ and $\left\|\beta_{\boldsymbol{w}}\right\|>0$. Let us prove that $\left\|\alpha_{w}^{\prime}\right\|^{2}\left\|\alpha_{g}\right\|^{2}+\left\|\beta_{w}^{\prime}\right\|^{2}\left\|\beta_{g}\right\|^{2}+1=Q$ must be satisfied by contradiction. Assume $\alpha_{w}^{\prime}$ and $\beta_{w}^{\prime}$ are the optimal complex weights for PD-2, which satisfy $\left\|\alpha_{w}^{\prime}\right\|^{2}\left\|\alpha_{g}\right\|^{2}+\left\|\beta_{w}^{\prime}\right\|^{2}\left\|\beta_{g}\right\|^{2}+1>Q$. Then, we can always find the special weights $\alpha_{w}^{*}$ and $\beta_{w}^{*}$ which satisfy:

$$
\left\{\begin{array}{l}
\left\|\alpha_{w}^{*}\right\|<\left\|\alpha_{w}^{\prime}\right\|, \\
\left\|\beta_{w}^{*}\right\|>\left\|\beta_{w}^{\prime}\right\|, \\
\left\|\alpha_{w}^{*}\right\|^{2}+\left\|\beta_{w}^{*}\right\|^{2}=\theta P, \\
\left\|\alpha_{w}^{*}\right\|^{2}\left\|\alpha_{g}\right\|^{2}+\left\|\beta_{w}^{*}\right\|^{2}\left\|\beta_{g}\right\|^{2}+1=Q .
\end{array}\right.
$$

Using Equation 89, and substituting $\alpha_{w}^{*}$ and $\beta_{w}^{*}$ into Equation 78, we obtain:

$$
\left\|\alpha_{\boldsymbol{w}}^{*}\right\|^{2}\|\boldsymbol{h}\|^{2}<\left\|\alpha_{\boldsymbol{w}}^{\prime}\right\|^{2}\|\boldsymbol{h}\|^{2}
$$

which contradicts the assumption that $\alpha_{w}^{\prime}$ and $\beta_{w}^{\prime}$ are the optimal complex weights for PD -2 . Combing the above discussions, at the optimal point, $\left\|\alpha_{w}\right\|^{2}\left\|\alpha_{g}\right\|^{2}+$ $\left\|\beta_{\boldsymbol{w}}\right\|^{2}\left\|\beta_{\boldsymbol{g}}\right\|^{2}+1=Q$ must always be satisfied.

Lemma 4 is thus proved.

Using lemma 2, lemma 3, and lemma 4, the closed-form solutions for $f_{1}(\theta)$ and $f_{2}(1-\theta)$ can be easily derived. To summarize, the following theorem is established.

Theorem 2. For any given $\theta$ that $(Q-1) /\left(P\|\boldsymbol{g}\|^{2}\right) \leq \theta \leq$ $(Q-1) /\left(P\left\|\left(\boldsymbol{I}-\overrightarrow{\boldsymbol{h}} \overrightarrow{\boldsymbol{h}}^{\mathrm{H}}\right) \boldsymbol{g}\right\|^{2}\right)$, the optimal energy beamforming vector for $f_{1}(\theta)$ can be derived as:

$$
\boldsymbol{w}^{*}=\alpha_{\boldsymbol{w}}^{*} \overrightarrow{\boldsymbol{h}}+\beta_{\boldsymbol{w}}^{*} \overrightarrow{\boldsymbol{h}}_{\perp}
$$

where $\eta_{w}^{*}$ and $\xi_{w}^{*}$ are the complex weights that can be expressed as:

$$
\begin{aligned}
& \alpha_{w}^{*}=\sqrt{\frac{Q-1-\theta P\left\|\beta_{g}\right\|^{2}}{\left\|\alpha_{g}\right\|^{2}-\left\|\beta_{g}\right\|^{2}}} \frac{\alpha_{g}}{\left\|\alpha_{g}\right\|}, \\
& \beta_{w}^{*}=\sqrt{\theta P-\frac{Q-1-\theta P\left\|\beta_{g}\right\|^{2}}{\left\|\alpha_{g}\right\|^{2}-\left\|\beta_{g}\right\|^{2}}} \frac{\beta_{g}}{\left\|\beta_{g}\right\|} .
\end{aligned}
$$

The optimal information beamforming vector for $f_{2}(1-\theta)$ can be derived as:

$$
\boldsymbol{s}^{*}=\sqrt{(1-\theta) P} \frac{\left(\boldsymbol{I}-\overrightarrow{\boldsymbol{g}} \overrightarrow{\boldsymbol{g}}^{\mathrm{H}}\right) \boldsymbol{h}}{\left\|\left(\boldsymbol{I}-\overrightarrow{\boldsymbol{g}} \overrightarrow{\boldsymbol{g}}^{\mathrm{H}}\right) \boldsymbol{h}\right\|} .
$$

where the proof is similarly as in $\mathbf{P B}-2$.

\section{Simulation results}

In this section, computer simulation results are presented to evaluate the performance of the proposed optimal approaches. Unless otherwise stated, it is assumed that the transmitter is equipped with $M=8$ antennas, and both the ID receiver and the $\mathrm{EH}$ receiver are equipped with single antenna. The entries of the channel vectors $\boldsymbol{h}$ and $\boldsymbol{g}$ are generated by independent circularly symmetric complex Gaussian (CSCG) random variables distributed as $\mathcal{C N}(0,1)$. The achievable rate $C$ is plotted versus a range of transmit power limit $P$ or harvested energy limit $Q$, where $P$ is defined in $\mathrm{dBm}$ and $Q$ is defined in $\mathrm{mW}$. The results are derived by averaging over 10,000 simulation trails.

In the first example, assuming the transmit power $P$ is constant, we can obtain the secret rate-energy region for the proposed algorithms.

In Figure 2, the transmit power is set to be $P=10 \mathrm{dBm}$. For the algorithm without using artificial noise, the secret rate monotonically decreases with the increasing of harvested energy $Q$, and will approach zero when $Q=18.5$ $\mathrm{mW}$. In practice, if the secret rate approaches zero, only the $\mathrm{EH}$ receiver can be satisfied, while the communication between the transmitter and the ID receiver should stop. For the algorithm with artificial noise (i.e., joint information and energy beamforming is used), the secret rate is much greater than that obtained by the algorithm without artificial noise. Interestingly, by the algorithm with artificial noise, the secret rate decreases slow when $Q \leq$ $15 \mathrm{~mW}$, while decreases fast when $Q>15 \mathrm{~mW}$, and approaches zero when $Q>20 \mathrm{~mW}$. The optimal power allocation ratio $\theta$ for the algorithm with artificial noise is demonstrated in Figure 3. It is clear that the optimal power allocation ratio $\theta$ increases with $Q$. When $Q=0$ 


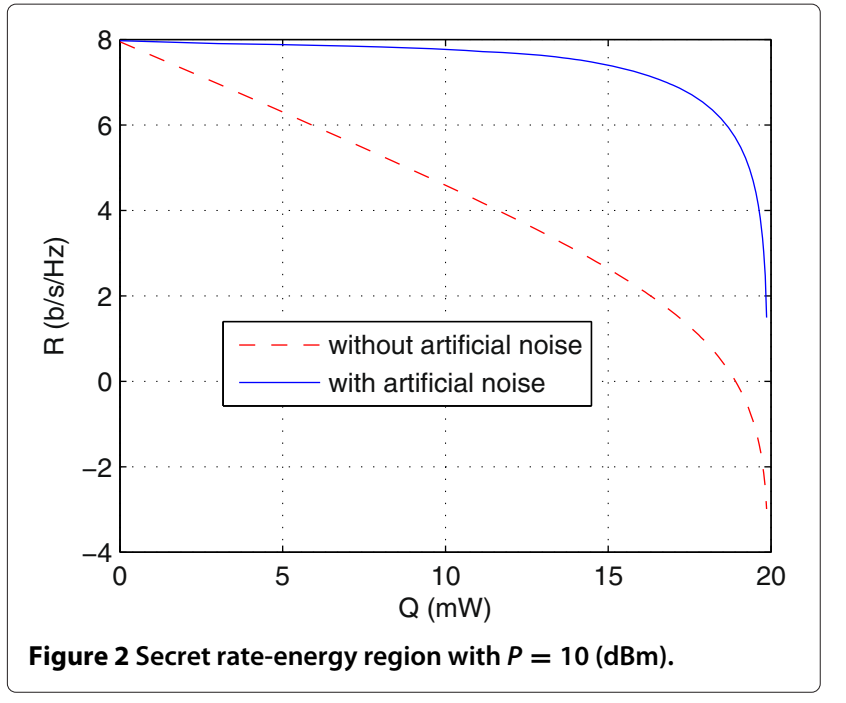

$\mathrm{mW}$, which means that there is no energy harvest requirement, no power should be used for energy beamforming. Thus, we obtain $\theta=0$. For the case that $Q=20 \mathrm{~mW}$, the optimal power allocation ratio $\theta$ is obtained as $\theta=$ 1 , which means that all power will be used for energy beamforming.

In the second example, assuming the harvested energy $Q$ is relatively constant, we can obtain the secret ratepower region for the proposed algorithms.

Assuming $Q$ is set to be $Q=Q_{\max } / 50$, the simulation results are demonstrated in Figure 4 . With the increase of $P$, the secret rate for both algorithms will monotonically increase with $P$ increasing. Remarkably, the secret rate obtained by the algorithm with artificial noise is always bigger than that obtained by the algorithm without artificial noise. The secret rate difference approaches $2 \mathrm{~b} / \mathrm{s} / \mathrm{Hz}$. Interestingly, the secret rate obtained by the algorithm

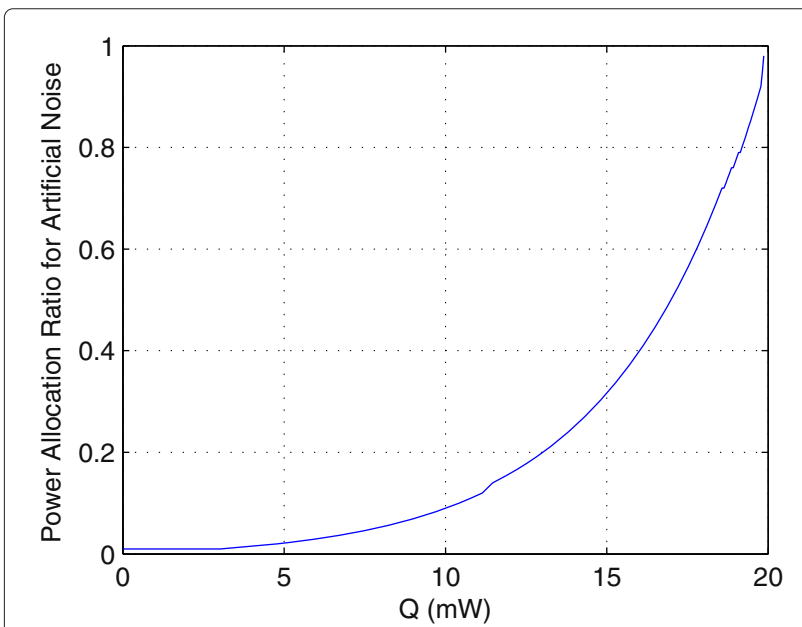

Figure 3 Optimal $\theta^{*}$ with $P=10(\mathrm{dBm})$.

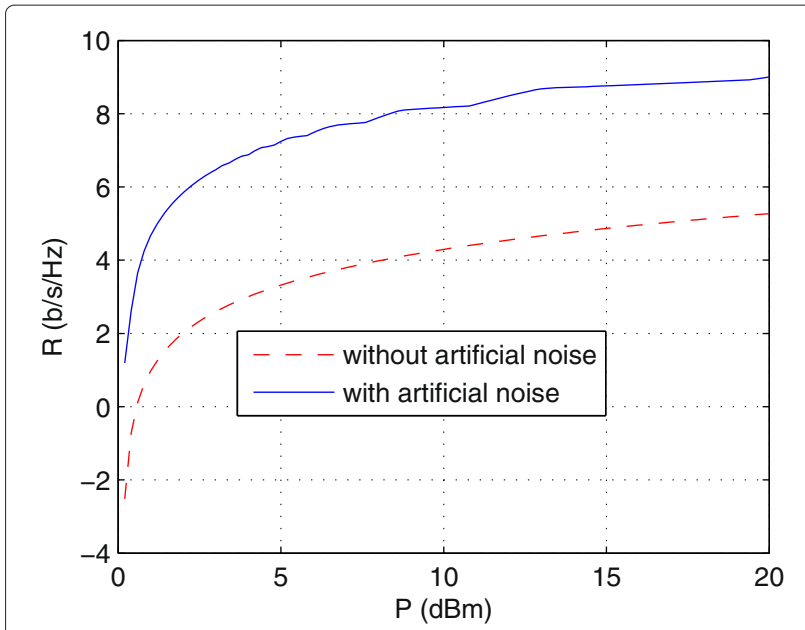

Figure 4 Secret rate-energy region with $Q=Q_{\max } / 50$.

with artificial noise increases fast when $P \leq 5 \mathrm{dBm}$. The reason lies in that when $P \leq 5 \mathrm{dBm}, \theta$ decreases fast, which is demonstrated in Figure 5.

\section{Conclusions}

This paper investigated the physical layer security for SWIPT. We designed zero-forcing-based joint information beamforming and energy beamforming to maximize the secret rate under the transmit power constraint and the energy harvesting constraint. The initial optimization was divided into subproblems for which closed-form solutions were obtain. The global optimal solution was also derived by using one-dimensional search. The conventional SWIPT without artificial noise was also studied, where closed-form solutions were also obtained. Simulation results were provided to evaluate the efficiency of the proposed algorithms.

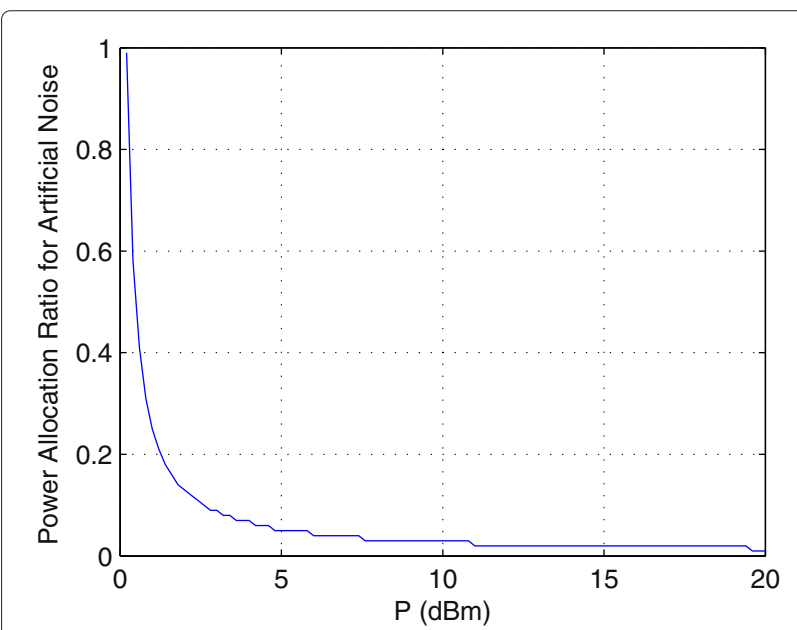

Figure 5 Optimal $\theta^{*}$ with $Q=Q_{\max } / 50$ 


\section{Endnotes}

${ }^{a}$ Note that the MSE-based design [29] could also be served as an interesting research topic.

${ }^{b}$ In general, the harvested energy is proportional to the energy of the received signal [4]. Hence, the harvested energy at the $\mathrm{EH}$ receiver can be computed from $Q=\zeta\left(|\boldsymbol{g s}|^{2}+|\boldsymbol{g} \boldsymbol{w}|^{2}\right)$, where $\zeta$ is a constant that accounts for the efficiency in the energy conversion. Interestingly, the energy limit $Q$ is very similar to the interference limit in cognitive radio (CR) [30-34], where the interference should be controlled to be below a predefined value.

\section{Competing interests}

The authors declare that they have no competing interests.

\section{Author details}

${ }^{1}$ Department of Automation, Tsinghua University, State Key Lab of Intelligent Technologies and Systems, Tsinghua National Laboratory for Information Science and Technology (TNList), No. 30 Shuang Qing Road, 100084 Beijing, P. R. China. ${ }^{2}$ High-Tech Institute of Xi'an, No. 2 Tong Xin Road, 710025 Xi'an, Shaanxi, P. R. China.

Received: 17 September 2014 Accepted: 9 February 2015 Published online: 10 March 2015

\section{References}

1. LR Varshney, Transporting information and energy simultaneously. Paper presented at the 2008 IEEE international symposium on information theory. IEEE, Toronto, ON, Canada, 6-11 July 2008

2. P Grover, A Sahai, Shannon meets tesla: wireless information and power transfer. Paper presented at the 2010 IEEE International Symposium on Information Theory. IEEE, Austin, TX, USA, 2010, pp. 2363-2367

3. Z Xiang, M Tao, Robust beamforming for wireless information and power transmission. IEEE Wireless Commun. Lett. 1(4), 372-375 (2012)

4. R Zhang, CK Ho, MIMO broadcasting for simultaneous wireless information and power transfer. IEEE Trans. Wireless Commun. 12(5), 1989-2001 (2013)

5. C Xing, N Wang, J Ni, Z Fei, J Kuang, MIMO beamforming designs with partial CSI under energy harvesting constraints. IEEE Signal Process. Lett. 20(4), 363-366 (2013)

6. AD Wyner, The wire-tap channel. Bell Syst. Tech. J. 54, 1355-1387 (1975)

7. I Csiszar, J Korner, Broadcast channels with confidential messages. IEEE Trans. Inf. Theory. 24(3), 339-348 (1978)

8. YC Liang, HV Poor, Generalized multiple access channels with confidential messages Paper presented at the 2006 IEEE international symposium on information theory. IEEE, Seattle, WA, USA, 9-14 July 2006, pp. 952-956

9. R Liu, I Maric, RD Yates, P Spasojevic, The discrete memoryless multiple access channel with confidential messages. Paper presented at the 2006 IEEE international symposium on information theory. IEEE, Seattle, WA USA, 9-14 July 2006, pp. 952-956

10. E Tekin, The Gaussian multiple access wire-tap channel: wireless secrecy and cooperative jamming. Paper presented at the 2006 information theory and applications workshop. IEEE, La Jolla, Canada, 2006, 29 January-2 February 2007, pp. 404-413

11. J Barros, MRD Rodrigues, Secrecy capacity of wireless channels. Paper presented at the 2006 information theory and applications workshop. IEEE, La Jolla, Canada, 9-14 July 2006, pp. 356-360

12. YC Liang, HV Poor, S Shamai, Secure communication over fading channels. IEEE Trans. Inf. Theory. 54(6), 2470-2492 (2008)

13. PK Gopala, L Lai, HE Gamal, On the secrecy capacity of fading channels. IEEE Trans. Inf. Theory. 54(10), 4687-4698 (2008)

14. R Negi, S Goel, Secret communication using artificial noise. Paper presented at the 2005 IEEE vehicular technology conference. IEEE, Dallas, Texas, USA, 25-28 September 2005, pp. 1906-1910

15. R Negi, S Goel, Guaranteeing secrecy using artificial noise. IEEE Trans. Wireless Commun. 7(6), 2180-2189 (2008)
16. A Khisti, GW Wornell, Secure transmission with multiple antennas I: the MISOME wiretap channel. IEEE Trans. Inf. Theory. 59(8), 3831-3842 (2010)

17. Z Xiangyun, MR McKay, Secure transmission with artificial noise over fading channels: achievable rate and optimal power allocation. IEEE Trans. Veh. Technol. 59(8), 3831-3842 (2010)

18. SAA Fakoorian, L Swindlehurst, Solutions for the MIMO gaussian wiretap channel with a cooperative jammer. IEEE Trans. Signal Process. 59(10), 5013-5022 (2011)

19. L Jiangyuan, AP Petropulu, KK Wong, Optimal cooperative jamming to enhance physical layer security using relays. IEEE Trans. Signal Process. 59(3), 1317-1322 (2011)

20. G Zheng, LC Choo, KK Wong, Optimal cooperative jamming to enhance physical layer security using relays. IEEE Trans. Signal Process. 59(3), 1317-1322 (2011)

21. H Jing, AL Swindlehurst, Robust secure transmission in MISO channels based on worst-case optimization. IEEE Trans. Signal Process. 60(4), 1696-1707 (2012)

22. S Gerbracht, C Scheunert, EA Jorswieck, Secrecy outage in MISO systems with partial channel information. IEEE Trans. Inf. Forenics Secur. 7(2), 704-716 (2012)

23. Q Li, WK Ma, Spatially selective artificial-noise aided transmit optimization for MISO multi-eves secrecy rate maximization. IEEE Trans. Signal Process. 61(10), 2704-2717 (2013)

24. WC Liao, TH Chang, WK Ma, Chi CY, QoS-based transmit beamforming in the presence of eavesdroppers: an optimized artificial-noise-aided approach. IEEE Trans. Signal Process. 59(3), 1202-1216 (2011)

25. M Pei, J Wei, KK Wong, X Wang, Masked beamforming for multiuser MIMO wiretap channels with imperfect CSI. IEEE Trans. Wireless Commun. 11(2), 544-549 (2012)

26. L Liu, R Zhangm, KC Chua, Secrecy wireless information and power transfer with MISO beamforming. IEEE Trans. Signal Process. 62(7), 1850-1863 (2014)

27. S Boyd, L Vandenberghe, Convex Optimization (Cambridge University Press, London, 2004)

28. S Shafiee, S Ulukus. Achievable rates in gaussian MISO channels with secrecy constraints. Paper presented at the 2007 IEEE international symposium on information theory (IEEE Nice, France, pp. 2466-2470

29. C Xing, S Li, Z Fei, J Kuang, How to understand linear minimum mean square error transceiver design for multiple input multiple output systems from quadratic matrix programming. IET Commun. 7(12), 1231-1242 (2013)

30. P Ren, Y Wang, Q Du, CAD-MAC:A channel-aggregation diversity based MAC protocol for spectrum and energy efficient conitive ad hoc networks. IEEE J. Sel. Areas Commun. 32(3), 237-250 (2014)

31. W Yin, P Ren, F Li, Q Du, Joint sensing and transmission for AF relay assisted PU transmission in cognitive radio networks. IEEE J. Sel. Areas Commun. 31(11), 2249-2261 (2013)

32. P Ren, Y Wang, Q Du, J Xu, A survey on dynamic spectrum access protocols for distributed cognitive wireless networks. Eurasip J. Wireless Commun. Netw. 2012(60), 1-21 (2012)

33. G Wu, P Ren, Q Du, Recall-based dynamic spectrum auction with the protection of primary users. IEEE J. Sel. Areas Commun. 30(10), 2070-2081 (2012)

34. TWang, L Song, Z Han, W Saad, Distributed cooperation radio networks: an overlapping coalition formation approach. IEEE Trans. Commun. 62(9), 3144-3160 (2014) 